University of Wollongong

Research Online

Faculty of Social Sciences - Papers (Archive) Faculty of Arts, Social Sciences \& Humanities

2018

Perceived stigma and social support in treatment for pharmaceutical opioid dependence

\author{
Sasha Cooper \\ University of New South Wales \\ Gabrielle Campbell \\ University of New South Wales \\ Briony K. Larance \\ University of Wollongong, blarance@uow.edu.au \\ Bridin Murnion \\ University of Sydney \\ Suzanne Nielsen \\ University of New South Wales
}

Follow this and additional works at: https://ro.uow.edu.au/sspapers

Part of the Education Commons, and the Social and Behavioral Sciences Commons

Research Online is the open access institutional repository for the University of Wollongong. For further information contact the UOW Library: research-pubs@uow.edu.au 


\title{
Perceived stigma and social support in treatment for pharmaceutical opioid dependence
}

\begin{abstract}
Introduction and Aims The dramatic increase in pharmaceutical opioid (PO) use in high-income countries is a growing public health concern. Stigma and social support are important as they may influence treatment uptake and outcomes, yet few studies exist regarding perceived stigma and social support among people with PO dependence. The aims of the study are to: (i) compare characteristics of those with PO dependence from iatrogenic and non-iatrogenic causes; (ii) document perceived stigma and its correlates in people in treatment for PO dependence; and (iii) examine correlates of social support in people in treatment for $\mathrm{PO}$ dependence. Design and Methods This prospective cohort study included $(\mathrm{n}=$ 108) PO-dependent people referred from treatment services. Telephone interviews were conducted at baseline, 3, 12 and 24 months. Multivariate linear regression was used to examine correlations. Results Mean age was $41(S D=10.5)$. Half $(n=56,52 \%)$ were female. Two in five met the criteria for iatrogenic dependence $(n=41,38 \%)$, with iatrogenic dependence associated with chronic pain, and no history of injection or heroin use. One quarter of study subjects reported past month unsanctioned opioid use $(n=$ $25,23 \%$ ). Being married/de facto or female was associated with higher levels of perceived stigma. Unsanctioned opioid use, iatrogenic dependence and mental health conditions were associated with lower social support. Discussion and Conclusions Stigma affects all people in treatment. Those who are married/de facto and female may benefit from interventions to address stigma. The association of low social support with poorer mental health and ongoing substance use indicate that treatment could focus more on this area.
\end{abstract}

\section{Keywords}

opioid, perceived, stigma, pharmaceutical, treatment, social, dependence, support

\section{Disciplines}

Education | Social and Behavioral Sciences

\section{Publication Details}

Cooper, S., Campbell, G., Larance, B., Murnion, B. \& Nielsen, S. (2018). Perceived stigma and social support in treatment for pharmaceutical opioid dependence. Drug and Alcohol Review, 37 (2), 262-272. 


\title{
Perceived Stigma and Social Support in Treatment for Pharmaceutical Opioid Dependence
}

\author{
Running Title: Stigma and social support in PO dependence \\ Sasha Cooper ${ }^{\mathrm{a}}$, Gabrielle Campbell ${ }^{\mathrm{b}}$, Briony Larance ${ }^{\mathrm{c}}$, Bridin \\ Murnion $^{\mathrm{d}} \&$ Suzanne Nielsen ${ }^{\mathrm{e}}$
}
a) Medical student, National Drug and Alcohol Research Centre (NDARC), University of New South Wales (UNSW), Sydney NSW 2052, Australia.
b) PhD. NHMRC Early Career Research Fellow, NDARC, UNSW, Sydney NSW 2052, Australia.
c) PhD. Senior Research Fellow, NDARC, UNSW, Sydney NSW 2052, Australia.
d) MBChB, FRACP, FFPMANZCA, FAChAM. Head of Department, Drug Health Services, Royal Prince Alfred Hospital, Sydney Local Health District, Camperdown, NSW, 2050. Clinical Senior Lecturer, Faculty of Medicine, University of Sydney, NSW 2006, Australia.
e) PhD. Senior Research Fellow, NDARC, UNSW, Sydney NSW 2052, Australia. Drug and Alcohol Services, South Eastern Sydney Local Health District, Surry Hills, NSW 2010 Australia.

\section{Corresponding author:}

Suzanne Nielsen

\section{Mailing address:}

National Drug and Alcohol Research Centre

University of New South Wales

Sydney NSW 2052

Australia

Telephone no: +61 (2) 89361017

Fax no: $+61(2) 93850222$

Email address: suzanne.nielsen@unsw.edu.au 


\begin{abstract}
Introduction: The dramatic increase in pharmaceutical opioid (PO) use in high-income countries is a growing public health concern. Stigma and social support are important as they may influence treatment uptake and outcomes, yet few studies exist regarding perceived stigma and social support among people with PO dependence.
\end{abstract}

Aims: To: (1) compare characteristics of those with PO dependence from iatrogenic and noniatrogenic causes; (2) document perceived stigma and its correlates in people in treatment for PO dependence; (3) examine correlates of social support in people in treatment for PO dependence.

Methods: Prospective cohort study $(n=108)$ of PO dependent people referred from treatment services. Telephone interviews were conducted at baseline, 3-, 12- and 24-months. Multivariate linear regression was used to examine correlations.

Results: Mean age was $41(S D=10.5)$. Half $(n=56,52 \%)$ were female. Two in five met the criteria for iatrogenic dependence $(n=41,38 \%)$, with iatrogenic dependence associated with chronic pain, and no history of injection or heroin use. One quarter reported past month unsanctioned opioid use $(n=25,23 \%)$. Being married/de facto or female was associated with higher levels of perceived stigma. Unsanctioned opioid use, iatrogenic dependence and mental health conditions were associated with lower social support.

Conclusions: Stigma affects all people in treatment. Those who are married/de facto and female may benefit from interventions to address stigma. The association of low social support with poorer mental health and ongoing substance use may indicate that treatment could focus more on this area.

\title{
Keywords
}

Analgesics, Opioid; Social Stigma; Social Support; Opioid-Related Disorders; Opioid Substitution Treatment.

\section{Conflict of Interest}

This research was funded by the Mental Health and Drug and Alcohol Research grants. Suzanne Nielsen (\#1013803, \#1132433), Briony Larance (\#1073858) and Gabrielle Campbell (\#1119992) are recipients of NHMRC research fellowships. The National Drug and Alcohol Research Centre at the University of NSW is supported by funding from the Australian Government under the Substance Misuse Prevention and Service Improvements Grant Fund. 


\section{Introduction}

Globally, pharmaceutical opioid (PO) utilisation has increased dramatically [1]. In Australia, PO dispensing where scripts were subsidised by the government rose from 500000 episodes in 1992, to 7.5 million in 2012 [2]. This increase is largely attributed to chronic pain treatment, despite little evidence for long-term effectiveness [3]. Alongside growing use, there are rising concerns regarding dependence, aberrant behaviour and overdose [4]. In Australia, accidental deaths due to any opioid increased by 76\% between 2002 and 2011 [2]. In both Australia and the US, PO-related mortality now exceeds heroin-related mortality [5, 6], with strong correlations between PO availability and related harms including mortality [7, 8].

Demand for medication-assisted (methadone or buprenorphine) opioid dependence treatment where a PO is the primary drug of concern is rising [9]. However, most treatment is informed by research where participants are dependent on heroin, and less is known about those who use PO. Although medication assisted treatments for opioid dependence are effective [10-12], to optimise treatment for people who are dependent on PO, it is critical to understand factors that may facilitate or deter treatment entry and support positive treatment outcomes. This is particularly timely in Australia, where changes in availability of over-the-counter (OTC) opioids in early 2018 [13] may contribute to treatment demand.

In the treatment of heroin dependence, greater social support is associated with better outcomes, such as earlier treatment-seeking [14], less heroin and other drug use [15-17], and improved mental health $[15,16]$. Enhancing medication-assisted treatment with social support services like counselling, psychiatric care and family therapy also results in further decreases in opioid use [18]. However, there have been no studies examining the impact of social support on treatment outcomes in people who are dependent on PO.

In addition to poor social support, stigma is likely to be an important barrier to treatment uptake and engagement in people dependent on PO [19]. Qualitative interviews with people who used OTC codeine found participants often referred to themselves as being 'addicted', and feeling "dirty and guilty," with dependence often hidden from family and friends [20]. They also held stigmatising attitudes towards those who used heroin, with employment, education and appearance listed as reasons why people who used codeine were more 'respectable'. Another study of people in treatment for OTC codeine dependence found there were perceptions that medication-assisted treatments for opioid dependence were for 'drug users', namely heroin, and so were for 'other people', thus separating themselves from illicit opioid users [19]. 
Stigma may also act as a barrier to pain relief, for example those dependent on opioids who request further pain relief may be seen as drug-seeking by physicians, leading to poor communication and under-treatment [21]. Additionally, those engaged in community pharmacy medication-assisted opioid dependence treatment may be subjected to suspicion and patronisation $[22,23]$. A study of people who used medications only as prescribed found the majority of participants believed that 'normal' people shouldn't have to be exposed to 'drug-takers' in the pharmacy [24]. These experiences suggest that stigma may pose an important barrier to treatment engagement for opioid dependent people, however this is not well researched in people dependent on PO.

Although social support has been more commonly examined, most research on both stigma and social support concerns only illicit substances, leaving large gaps in the literature regarding PO dependence. This study utilises data from a cohort study conducted over two years with people in treatment for PO dependence. It examines perceived stigma and social support, which are modifiable factors that may improve treatment entry and outcomes for this emerging population. The aims of this study are:

1. To compare characteristics of those with PO dependence from iatrogenic and noniatrogenic causes.

2. To document perceived stigma and its correlates in people in treatment for PO dependence.

3. To examine correlates of social support in people in treatment for PO dependence.

\section{Methods}

This study utilises baseline, 3-, 12- and 24-month follow-up data collected from a cohort of people in treatment for PO dependence. Ethics approval was obtained from Royal Prince Alfred Hospital Human Research Ethics Committee (HREC), and HRECs for the six Local Health Districts.

\section{Participants}

This study represents one component of a larger study [25]. There were 108 participants recruited for the parent study via healthcare provider referral or advertisements in drug treatment centres within New South Wales. Eligibility criteria included those who had entered treatment for PO dependence, and who were competent in English. Interviews were 
conducted via telephone at baseline, 3-, 12- and 24 months. Baseline interviews ranged from 45 minutes to 1.5 hours in duration. Subsequent interviews ranged from 30 minutes to 1 hour. Participants were reimbursed $\$ 40$ after each interview.

\section{Measures}

\section{Demographic Measures}

Age and gender were collected. Education level was categorised into 'completed year 10 or less', 'completed year 12/TAFE/technical college' and 'completed university/college'. Employment was dichotomised into 'employed/student' and 'not employed' to retain power for analyses. Marital status was dichotomised into 'married/de facto' and 'not married'. Participants were asked who they currently lived with, which was dichotomised into 'living alone' and 'living with others'.

\section{Physical Health}

Current health rating was determined using one item from the SF-12 Health Survey [26]: "In general, would you say your health is....?”. This was dichotomised into 'excellent/very good/good' and 'fair/poor'. Participants were also asked about problematic arthritis or rheumatism, chronic back or neck problems, frequent or severe headaches, visceral pain, generalised pain and other chronic pain conditions in the past 12 months. This was dichotomised into 'current chronic pain' or 'no current chronic pain'.

\section{Opioid Use History}

Participants were asked about their current treatment length, and which pharmaceutical opioid led them to treatment. 'Primary opioid of concern' was then dichotomised into 'strong opioids' (schedule 8: morphine, oxycodone, methadone, buprenorphine, fentanyl, hydromorphone) or 'weak opioids' (schedule 3 or 4: codeine, tramadol). Participants were asked about their first source ("When you first started to have a problem with taking more opioid analgesics than prescribed or misusing opioid analgesics, what was your first source?") and reason for opioid use ("what was the major reason you first used opioid analgesics?"). They were classified as having 'iatrogenic dependence' if their first opioids of concern were prescribed by a doctor for a legitimate medical reason.

Past month medical and illicit opioid use was reported, and 'unsanctioned opioid use in the past month' was defined as non-medical use of any opioids, or use of heroin. Participants 
were also asked, "have you ever used heroin?" and whether they had ever injected heroin, non-medicinal or non-prescribed opioids, methamphetamine, cocaine, ecstasy, cannabis, alcohol, tobacco, non-medicinal or non-prescribed benzodiazepines, hallucinogens or other. This was dichotomised into 'have injected' and 'have never injected'.

\section{Mental Health}

Participants were asked to report if they had been diagnosed with a range of mental health conditions including depression, dysthymia, anxiety/panic disorder, bipolar/manic depressive disorder, obsessive-compulsive disorder, schizophrenia, psychosis, ADD/ADHD and personality disorder, and to report if these had been problematic in the past 12 months. Additionally a cut-off score of $\geq 3$ on the Primary Care PTSD screen [27] was considered indicative of post-traumatic stress disorder. All conditions were summed to create a 'mental health conditions in past 12 months' variable.

\section{Perceived Stigma of Addiction Scale (PSAS)}

This scale contains 8 items scored between 1 (strongly disagree) and 4 (strongly agree) (Appendix A) [28]. Participants were asked to complete this survey in the 24-month interview. Questions 1, 2, 3, 4, 6 and 8 were reverse scored. Item scores were combined to obtain a total score between 8 and 32, where higher scores indicated more perceived stigma. This scale has been found to display good convergent and discriminant validity, and adequate internal consistency reliability $(\alpha=.73)$ [28]. It has previously been used in studies concerning opioid dependence [29] and substance use more generally [30].

\section{Medical Outcomes Study (MOS) Social Support Survey}

This survey contains items that ask how often support is available and is scored between 1 (none of the time) and 5 (all of the time) [31]. It contains 19 items sorted into 5 scales: (1) An emotional/informational subscale, (2) a positive interaction subscale, (3) a tangible support subscale, (4) an affectionate support subscale and (5) a combined overall social support scale [31]. Total scores are calculated with a provided formula [32], and range from 0 to 100 , where higher scores indicate more support. For the purpose of this study we used only the items that formed the emotional/informational support (EIS) and positive interaction support (PIS) subscales, and the overall support (OS) score (the sum of all questions) (Appendix B). This survey has been found to display high convergent and discriminant validity, and high internal consistency reliability $(\alpha=.97)$ [31]. It has been used in previous research with 
people prescribed PO for chronic non-cancer pain [33], and those in medication-assisted treatment for any opioid use [34].

\section{Analysis}

Cronbach's alpha was calculated for stigma and social support scales. The internal consistency reliability of the PSAS was acceptable $(\alpha=.744)$, and for the MOS social support survey was excellent $(\alpha=.965)$.

Analyses were conducted using SPSS version 22.0. Chi-square tests, Independent t-tests and Mann-Whitney $U$ tests were used to examine baseline differences between those who initiated opioid use for iatrogenic and non-iatrogenic reasons. Bivariate correlates of stigma and social support were determined using Independent t-tests, ANOVA and Pearson's product-moment correlations where variables were normally distributed. Where they were not normally distributed, Mann-Whitney U tests, Kruskal-Wallis tests and Spearman's correlations were used. Multivariate linear regression was then used to examine significant bivariate associations after controlling for age, gender and other variables significant at the bivariate level. Generalised estimating equations (GEE) were used to determine changes in overall social support over the 2-year study period.

To determine effects of attrition on the data, participants who completed all four interviews $(n=85)$, and those who completed between one and three interviews $(n=23)$ were compared. There were no significant differences (Appendix D).

There was a large amount of missing stigma data (missing $n=21 / 87$ ), as a total score could not be calculated with a single question unanswered. As the scale includes no instructions for missing data, we used multiple imputation with fully conditional specification to impute missing item-level data to maintain power for our analysis. As $24 \%$ of the data was missing, 20 imputations were run [35]. Age, sex, education, employment, living situation, marital status, health rating, iatrogenic dependence, primary drug of concern, heroin use history, injection history, OS, EIS, PIS, and completed stigma questions were used as predictors. Analyses were performed with both complete-case data and imputed data, with correlates deemed dependable if they were significant with imputed data, and showed an association in the same direction with complete-case data. 


\section{Results}

\section{Baseline characteristics according to whether initiation of $\mathrm{PO}$ use was iatrogenic or non-iatrogenic}

The mean age was 41 years $(S D=10.54)$. Half $(n=56,52 \%)$ were female and one third ( $n=$ $32,30 \%)$ reported current employment/study (Table 1$)$. One third $(n=37,34 \%)$ were married/de facto, and most $(n=73,68 \%)$ were living with others. Forty-one participants (38\%) reported current chronic pain, and 38\% $(n=41)$ were classified as having iatrogenic dependence. Just over half $(n=61,57 \%)$ had ever used heroin, and a quarter had used unsanctioned opioids within the past month $(n=25,23 \%)$.

Iatrogenic dependence was associated with a higher prevalence of chronic pain, compared to non-iatrogenic dependence ( $56 \%$ vs. $27 \%$, OR $3.48,95 \%$ CI $[1.53,7.90])$. It was also associated with a lower prevalence of lifetime heroin use ( $40 \%$ vs. $67 \%$, OR $0.31,95 \% \mathrm{CI}$ $[0.14,0.70])$, and injection of any drug ( $42 \%$ vs. $69 \%$, OR $0.32,95 \%$ CI $[0.14,0.73])$ (Table 1).

\section{Perceived Stigma}

Participants reported that they perceived stigma in all domains (including childcare, employment and personal interaction), scoring a mean of 23.30 out of a possible range of 8 to 32 (Table 1). Perceptions of stigma were greatest around the prospect of caring for children and gaining employment, and least around friendship and dating, though scores still indicated stigma in these domains (Figure 1) (Appendix A).

Bivariate analysis identified that being married or in a de facto relationship and being female were associated with higher levels of overall perceived stigma (Table 2). Both genders experienced stigma in similar domains, but females consistently reported more stigma for each question, though the differences were not significant in any individual domain (Figure 1). After controlling for age and gender in a multivariate linear regression model, only marital status remained significantly associated with higher levels of overall perceived stigma ( $B=$ $2.00,95 \% \mathrm{CI}[0.46,3.54] p=.011$ ) (results not presented).

\section{Social Support}

The median overall support (OS) score was $54.17(I Q R=47.40$, scale $0-100)$, median 
emotional/informational support (EIS) score was $62.50(I Q R=43.75)$ and median positive interaction support (PIS) score was $58.33(I Q R=50.00)$. Initially associations of social support were to be examined longitudinally, however no significant effect of time on OS was identified (i.e. social support did not change over time) (Appendix C), so cross sectional analyses at Baseline are presented, providing the largest sample size to explore associations.

At the bivariate level, unsanctioned opioid use and the number of mental health conditions were associated with lower levels of OS, EIS and PIS (Table 4). Iatrogenic dependence was associated with lower OS and EIS, but did not reach significance for PIS. Length of current treatment episode was negatively associated only with PIS. Living with other people and being married or in a de facto relationship was associated with higher OS, EIS and PIS. Being employed or studying correlated positively with only PIS.

Controlling for age, gender and variables significant at the bivariate level, unsanctioned opioid use in the past month remained significant for lower levels of OS $(B=-19.36,95 \% \mathrm{CI}$ $[-31.10,-7.31] p=.002)$, EIS $(B=-21.93,95 \%$ CI $[-34.26,-9.60] p=.001)$ and PIS $(B=-$ $14.71,95 \%$ CI $[-28.25,-1.16] p=.034)$. Mental health conditions remained significantly associated with lower OS $(B=-3.83,95 \%$ CI [-6.77, -0.89] $p=.011)$, EIS $(B=-3.44,95 \%$ CI $[-6.46,-0.43] p=.026)$ and PIS $(B=-3.73,95 \%$ CI $[-7.11,-0.34] p=.031)$. Iatrogenic dependence remained significantly associated with lower OS $(B=-12.62,95 \%$ CI [-22.73, $2.50] p=.015)$ and EIS $(B=-12.93,95 \%$ CI $[-23.21,-2.65] p=.014)$ (results not presented).

\section{Discussion}

This is the first study to systematically explore perceived stigma and social support in a cohort of people in treatment for PO dependence. Participants reported considerable levels of perceived stigma in all domains examined, with the highest levels relating to childcare and employment. One possible explanation for this is great levels of perceived responsibility, reflecting a societal protectiveness of children and condemnation of exposing them to risktaking behaviour, as well as the importance of performing jobs safely and effectively. Unsanctioned opioid use, mental health conditions and dependence from iatrogenic opioid use were associated with lower levels of social support. This suggests that a greater focus on increasing social support in treatment may be warranted. 
Being married or in a de facto relationship was the strongest independent predictor of higher perceived stigma. This finding may appear counterintuitive, as the individual scale question "most people would be willing to date someone who has been in treatment for substance use", was scored lowest by the cohort, indicating they saw relationships as the least stigmatising scenario. This discrepancy may highlight complex interactions between stigma and relationships. One strategy to address stigma in married people may be involvement of drug-free partners in treatment, in an attempt to expose them to reasons behind their partner's drug use, and provide education that dependence is a medical condition that may be treated [36].

High levels of perceived stigma may act as a barrier to treatment engagement. National drug control strategies often revolve around strict law enforcement and depictions of drug-related harm in the media, attempting to discourage people from drug use. However, depictions of negative images related to drug use increases social stigma, whilst decreasing public support for effective treatment in favour of punishment [37]. Importantly, shame amongst people in drug treatment has been linked with relapse [38]. To target stigma at a societal level, it may be useful to depict successfully treated drug dependence [37], and implement community awareness campaigns and education programs aimed at professionals including police, doctors and counsellors [39].

Several factors including heroin use history, injection history and mental health problems were not associated with differences in perceived stigma. These findings are of importance, as Australian and UK studies of people who used OTC codeine demonstrated stigmatising attitudes towards people who used heroin or injected drugs [19, 20]. Our finding of no association between heroin use or injection with higher levels of stigma suggest that the way people in treatment for PO use perceive stigma towards themselves may be different to how they project it on others.

Unsanctioned opioid use in the past month, mental health conditions and iatrogenic dependence were independently associated with lower levels of social support. Studies have shown that higher levels of social support [15], and specific social network interventions [40] may lead to better treatment outcomes in opioid dependent people. Our results indicate that those with unsanctioned opioid use, mental health conditions and iatrogenic dependence may benefit most from interventions to increase social support, given the positive associations between social support and treatment outcomes. Future work examining social network involvement in all facets of treatment, for example pain management, mental health counselling and opioid treatment, could test if interventions to improve social support may 
benefit those with PO dependence.

Our findings of lower social support in those with iatrogenic dependence are in keeping with previous studies of social support in chronic pain. In qualitative interviews of people with chronic pain, only two out of thirteen participants reported that they had someone they could talk to in depth about their experience [41]. This is important, as social support may have beneficial effects on morbidity, mortality and quality of life in chronic pain populations [42$44]$, in addition to potential positive effects on substance dependence treatment outcomes [15, 40].

The results of this study indicate that there was no change in social support over time. This may have been due to the social support measure lacking sensitivity in this population. The sensitivity has not been measured in PO dependent people. However, the scale has previously detected significant longitudinal changes in other studies [45]. Only those who had already entered treatment were recruited, but it may be the case that greater changes in social support are observed alongside uptake of treatment, which may not have been captured due to this eligibility criterion. Additionally, changes in social support after entering treatment may take longer to observe than the study length of two years. As such, future work may include a longer follow-up period. Another explanation for these findings may relate to the treatment itself. As standard treatment practices generally do not involve family, friends or partners, changes in social support may not be expected. The finding that social support did not appear to change on its own indicates that social interventions may be required to increase support, in an attempt to improve treatment outcomes.

Some limitations should be considered in interpreting these findings. We recruited participants already engaged in treatment through advertisements and healthcare provider referral. This method may have excluded those who felt most stigmatised, who may not have entered treatment or agreed to be referred for the study. All data was obtained via self-report, which is inherently subject to reporting bias, however self-report studies are generally considered reliable in the context of substance abuse [46]. The use of multiple imputation to obtain complete stigma data introduced inherent limitations to the final results. These were minimised through the number of imputations, and running analyses with both case-complete and imputed data. Additionally as the stigma scale was only included in the 24-month interview, changes in stigma over time could not be examined. Although of modest sample size, this study is the first of its kind, lending importance to its findings. There was a good retention rate at the 2-year interview $(80 \%)$ and there were no significant differences between those who completed one to three interviews, and those who completed all four interviews, 
suggesting attrition may not have influenced results.

\section{Conclusions}

This study represents the first in Australia to examine perceived stigma and social support in a cohort of people in treatment for PO dependence, an area that has been neglected in the literature. Overall, the findings of this study identify that women and people in a relationship may be most affected by stigma, whilst those who exhibit unsanctioned opioid use, mental health conditions and iatrogenic dependence may benefit most from social support interventions. Future studies might build on these findings, aiming to address stigma and social support to improve treatment uptake and outcomes in people dependent on PO.

\section{Acknowledgements}

We wish to acknowledge investigators for the parent study: Raimondo Bruno, Nicholas Lintzeris, Michael Vanderhaven, Gina Lattas, Simon Holliday, Stephen Ling, Craig Sadler, Rohan Holland, Adrian Dunlop, Catherine Silsbury, Paul Haber, Bridin Murnion, Antonia Hordern, Mark Hardy, Apo Demirkol, Lucy Burns, Gabrielle Campbell, Jenny Houseman and Louisa Degenhardt.

Participants were recruited with the help of David Helliwell, Jennifer Johnston, Amanda Brown, Rohan Holland, Simon Holliday, Craig Sadler, Stephen Ling, Adrian Dunlop, Glenys Dore, Mark Hardy, Jennie Houseman, Nicholas Lintzeris, Apo Demirkol, Carolyn Smith, Stefanie Leung, Bridin Murnion, Paul Haber, Catherine Silsbury, Nghi Phung, Trish Collie, Andrew Hallinan, and staff at the Kobi Clinic, Garden Court Clinic and Scott Street Clinic.

Antonia Hordern, Michael Vanderhaven, Gina Lattas and Lexi Myers assisted with data collection. Statistical assistance was provided by Philip Clare.

\section{References}

1. Hastie BA, Gilson AM, Maurer MA, Cleary JF. An examination of global and regional opioid consumption trends 1980-2011. Journal of Pain \& Palliative Care Pharmacotherapy. 2014;28(3):25975 . 
2. Blanch B, Pearson SA, Haber PS. An overview of the patterns of prescription opioid use, costs and related harms in Australia. British Journal of Clinical Pharmacology. 2014;78(5):1159-66.

3. Kissin I. Long-term opioid treatment of chronic nonmalignant pain: unproven efficacy and neglected safety? Journal of Pain Research. 2013;6:513-29.

4. Ling W, Mooney L, Hillhouse M. Prescription opioid abuse, pain and addiction: clinical issues and implications. Drug and Alcohol Review. 2011;30(3):300-5.

5. Paulozzi LJ, Xi Y. Recent changes in drug poisoning mortality in the United States by urbanrural status and by drug type. Pharmacoepidemiology \& Drug Safety. 2008;17(10):997-1005.

6. Roxburgh A, Burns L. Accidental drug-induced deaths due to opioids in Australia, 2011. Sydney: National Drug and Alcohol Research Centre. 2015.

7. Rintoul AC, Dobbin MDH, Drummer OH, Ozanne-Smith J. Increasing deaths involving oxycodone, Victoria, Australia, 2000-09. Injury Prevention. 2010;17(4):254-9.

8. Fischer B, Jones W, Rehm J. High correlations between levels of consumption and mortality related to strong prescription opioid analgesics in British Columbia and Ontario, 2005-2009.

Pharmacoepidemiology and drug safety. 2013;22(4):438-42.

9. Nielsen S, Roxburgh A, Bruno R, Lintzeris N, Jefferson A, Degenhardt L. Changes in nonopioid substitution treatment episodes for pharmaceutical opioids and heroin from 2002 to 2011. Drug \& Alcohol Dependence. 2015;149:212-9.

10. Mattick RP, Breen C, Kimber J, Davoli M. Buprenorphine maintenance versus placebo or methadone maintenance for opioid dependence. Cochrane Database of Systematic Reviews. 2014(2). 11. Nielsen S, Larance B, Degenhardt L, Gowing L, Kehler C, Lintzeris N. Opioid agonist treatment for pharmaceutical opioid dependent people. Cochrane Database of Systematic Reviews. 2016(5).

12. Nielsen S, Larance B, Lintzeris N. Opioid agonist treatment for patients with dependence on prescription opioids. JAMA. 2017;317(9):967-8.

13. TGA. Proposal for the re-scheduling of Codeine products 2015 [Available from:

https://www.tga.gov.au/media-release/proposal-re-scheduling-codeine-products.

14. Kelly SM, O'Grady KE, Schwartz RP, Peterson JA, Wilson ME, Brown BS. The relationship of social support to treatment entry and engagement: the Community Assessment Inventory. Subst Abus. 2010;31(1):43-52.

15. Warren JI, Stein JA, Grella CE. Role of social support and self-efficacy in treatment outcomes among clients with co-occurring disorders. Drug \& Alcohol Dependence. 2007;89(2-3).

16. Costantini M, Wermuth L, Sorensen J, Lyons J. Family functioning as a predictor of progress in substance abuse treatment. Journal of Substance Abuse Treatment. 1992;9(4):331-5.

17. Day E, Copello A, Karia M, Roche J, Grewal P, George S, et al. Social network support for individuals receiving opiate substitution treatment and its association with treatment progress.

European Addiction Research. 2013;19(4):211-21.

18. McLellan AT, Arndt IO, Metzger DS, Woody GE, O'Brien CP. The effects of psychosocial services in substance abuse treatment. JAMA. 1993;269(15):1953-9.

19. Nielsen S, Cameron J, Pahoki S. Over the counter codeine dependence. Melbourne: Turning Point Alcohol and Drug Centre; 2010.

20. Cooper RJ. 'I can't be an addict. I am.' Over-the-counter medicine abuse: a qualitative study. BMJ Open. 2013;3(6).

21. Esquibel AY, Borkan J. Doctors and patients in pain: Conflict and collaboration in opioid prescription in primary care. Pain. 2014;155(12):2575-82.

22. Harris J, McElrath K. Methadone as social control: institutionalized stigma and the prospect of recovery. Qual Health Res. 2012;22(6):810-24.

23. Anstice S, Strike CJ, Brands B. Supervised methadone consumption: client issues and stigma. Substance Use \& Misuse. 2009;44(6):794-808.

24. Gidman W, Coomber R. Contested space in the pharmacy: public attitudes to pharmacy harm reduction services in the West of Scotland. Res Social Adm Pharm. 2014;10(3):576-87.

25. Nielsen S, Larance B, Bruno R, Lintzeris N, Vanderhaven M, Lattas G, et al. Technical Report 331: Understanding the health and service needs of diverse populations of pharmaceutical opioid users: Cohort studies of dependent users in treatment, and people who inject drugs. National Drug and Alcohol Research Centre; 2015.

26. Ware JE, Kosinski M, Keller SD. A 12-item short-form health survey: construction of scales and preliminary tests of reliability and validity. Medical Care. 1996;34(3):220-33. 
27. Prins A, Ouimette P, Kimerling R, Cameron RP, Hugelshofer DS, Shaw-Hegwer J, et al. The primary care PTSD screen (PC-PTSD): Development and operating characteristics Primary Care Psychiatry. 2003;9(1):9-14.

28. Luoma JB, O'Hair AK, Kohlenberg BS, Hayes SC, Fletcher L. The development and psychometric properties of a new measure of perceived stigma toward substance users. Substane Use \& Misuse. 2010;45(1-2):47-57.

29. Mattoo SK, Sarkar S, Gupta S, Nebhinani N, Parakh P, Basu D. Stigma Towards Substance use: Comparing Treatment Seeking Alcohol and Opioid Dependent men. International Journal of Mental Health and Addiction. 2014;13(1):73-81.

30. Stringer K, Schumacher JE, Turan JM, Mugavero M, Kempf MC, Baker E. The perceived stigma of substance abuse scale: Reliability and validity with substance using people living with HIV. Drug and Alcohol Dependence. 2015;156:e215.

31. Sherbourne CD, Stewart A. The MOS social support survey. Social Science \& Medicine. 1991;32(6):705-14.

32. RAND Health. Social support survey instrument scoring instructions 2016 [Available from: http://www.rand.org/health/surveys_tools/mos/social-support/scoring.html.

33. Campbell G, Nielsen S, Bruno R, Lintzeris N, Cohen M, Hall W, et al. The Pain and Opioids IN Treatment study: characteristics of a cohort using opioids to manage chronic non-cancer pain. Pain. 2015;156(2):231-42.

34. Wasserman DA, Stewart AL, Delucchi KL. Social support and abstinence from opiates and cocaine during opioid maintenance treatment Drug \& Alcohol Dependence. 2001;65(1):65-75.

35. Graham JW, Olchowski AE, Gilreath TD. How many imputations are really needed? Some practical clarifications of multiple imputation theory. Prevention Science. 2007;8(3):206-13.

36. Woods JS, Joseph H. Reducing stigma through education to enhance Medication-Assisted Recovery. Journal of Addictive Diseases. 2012;31(3):226-35.

37. McGinty EE, Goldman HH, Pescosolido B, Barry CL. Portraying mental illness and drug addiction as treatable health conditions: effects of a randomized experiment on stigma and discrimination. Social Science \& Medicine. 2015;126:73-85.

38. Randles D, Tracy JL. Nonverbal displays of shame predict relapse and declining health in recovering alcoholics. Clinical Psychological Science. 2013;1(2):149-55.

39. Livingston JD, Milne T, Fang ML, Amari E. The effectiveness of interventions for reducing stigma related to substance use disorders: a systematic review. Addiction. 2012;107(1):39-50.

40. Galanter M, Dermatis H, Glickman L, Maslansky R, Sellers M, Neumann E, et al. Network therapy: decreased secondary opioid use during buprenorphine maintenance. Journal of Substance Abuse Treatment. 2004;26(4):313-8.

41. Thomas SP. A phenomenologic study of chronic pain. Western Journal of Nursing Research. 2000;22(6):683-705.

42. Montoya P, Larbig W, Braun C, Preissl H, Birbaumer N. Influence of social support and emotional context on pain processing and magnetic brain responses in fibromyalgia. Arthritis \& Rheumatology. 2004;50(12):4035-44.

43. Franks HM, Cronan TA, Oliver K. Social support in women with fibromyalgia: Is quality more important than quantity? Journal of Community Psychology. 2004;32(4):425-38.

44. Pitsilka DA, Kafetsios K, Niakas D. Social support and quality of life in patients with rheumatoid arthritis in Greece. Clinical and Experimental Rheumatology. 2015;33(1):27-33.

45. Holden L, Dobson AJ, Ware RS, Hockey R, Lee C. Longitudinal trajectory patterns of social support and associated mental health in an Australian cohort of young women. Quality of Life Research. 2015;24(9):2075-86.

46. Darke S. Self-report among injecting drug users: a review. Drug \& Alcohol Dependence. 1998;51(3):253-63. 